\title{
Erythrocyte membrane in the evaluation of neurodegenerative disorders
}

This article was published in the following Dove Press journal:

Degenerative Neurological and Neuromuscular Disease

17 October 2017

Number of times this article has been viewed

\author{
Anjana Sadanand' \\ Anjali Janardhanan' \\ Arun Sankaradoss' \\ Arambakkam J Vanisree' \\ Thamilpavai Arulnambi ${ }^{2}$ \\ Kesavamurthy Bhanu ${ }^{2}$ \\ 'Department of Biochemistry, \\ University of Madras, ${ }^{2}$ Department of \\ Neurology and Neurosurgery, Madras \\ Medical College, Chennai, India
}

Correspondence: Arambakkam J Vanisree Department of Biochemistry, University of Madras, Guindy Campus, 9/36,

Gandhi Mandapam Road, Chitra Nagar,

Kotturpuram, Chennai 600025, India

Tel +9l 04422202735

Email journalsvuom@gmail.com

\begin{abstract}
Neurodegenerative diseases have many similar pathological conditions, and very few studies exist which detail their molecular features. Proteins like $\mathrm{Na}^{+} / \mathrm{K}^{+}$-ATPase (NKA), $\alpha$-spectrin (SPTA) and drebrin have been reported to be involved in the integrity of neuronal cell membrane and their functions. Furthermore, recent studies have highlighted their implication in neurodegeneration. In the current study, we wanted to identify the role of NKA, SPTA and drebrin in the erythrocyte membranes obtained from the blood of patients with neurodegenerative disorders (NDs) subjected to motor impairment such as Parkinson's disease, amyotrophic lateral sclerosis, ataxia and dementia. We have studied the activity of NKA and the expression of NKA, SPTA and drebrin in the erythrocyte membrane by quantitative real-time PCR and Western blot obtained from the blood samples of patients with NDs culminating in movement and memory dysfunction. We observed a significant reduction in the expressions of NKA, SPTA and drebrin when compared to control and significant variations among the recruited ND samples. On correlating, we found a significant relationship between the expressions and the clinical features such as bradykinesia. Thus, we suggest that the reduction in the expressions of NKA, SPTA and drebrin could function as tools of assessment and speculate the particular neurodegenerative condition.
\end{abstract}

Keywords: $\mathrm{Na}^{+} / \mathrm{K}^{+}$-ATPase, spectrin, drebrin, neurodegeneration and movement disorder

\section{Introduction}

Neurodegenerative disorders (NDs) are a complex group of disorders characterized by neural degeneration in various regions of brain and neurons. Diagnosis of NDs are based mainly on the clinical features and are difficult to identify based on a particular condition. Bradykinesia or slowness in movement is one of the prime features of movement disorders ${ }^{1}$ like Parkinson's disease (PD), amyotrophic lateral sclerosis (ALS) and ataxia. The Modified Bradykinesia Rating Scale (MBRS) is used to identify bradykinesia and measure the motor deficits in neurodegeneration, which provides an insight into neurophysiological investigation for clinicians to apprehend alterations of motor activity or motor excitability. ${ }^{2}$ The pathophysiology that underlies this feature is subjected to various views such as failure in basal ganglia output to reinforce cortical mechanism. ${ }^{3}$ There have been vast studies in identifying parameters of neurotransmission and biochemical characteristics, similar to the central nervous system, in platelets, erythrocytes and lymphocytes in search of peripheral markers that can identify degeneration and its progression in human brain. ${ }^{4}$ All these biomarkers are machinery of an intertwined biological system, wherein functional and structural 
characteristics of the erythrocyte membrane act as a sensor of pathological changes. ${ }^{5}$ We attempted to study the role of erythrocyte membrane through biochemical modes in NDs (PD, ALS, ataxia and dementia).

$\mathrm{Na}^{+} / \mathrm{K}^{+}$-ATPase (NKA) is important in maintaining intracellular gradients of ions in cells. NKA plays a crucial role in cellular metabolism as it accounts for $\sim 30 \%$ of the total body energy consumption and for $50 \%$ of the brain energy consumption. ${ }^{5}$ There have been reports that highlighted the reduction in the activity of NKA in erythrocytes of patients with neurological disorders which predisposes its role in these diseases. ${ }^{6}$

Spectrins are enriched membrane-associated molecules which were discovered in human red blood cells and further ubiquitously identified in various other bodily tissues including the brain. These are the integral proteins of the erythrocyte membranes along with other integral membrane proteins; in association with actin-like proteins, they form a two-dimensional meshwork and provide elasticity, flexibility and stability against shear stress for the passage of erythrocyte membranes in the circulatory system. ${ }^{7}$ They are engaged mainly in the organization and function of membrane integrity. ${ }^{8}$ Alpha-spectrin (SPTA) is usually linked to the cytoskeleton and is involved in membrane maintenance, thereby these molecules have important biological functions in the brain during neurodevelopment and synaptoplacticity. ${ }^{9}$ Drebrin is another protein that contributes equally in cell shape organizations..$^{10}$ It is an actin-bound protein that helps in bundling protein in cytoskeleton formation. There are many studies on drebrin expression in neurons ${ }^{11}$ and are found in other cell types including lymphocytes, endothelial cells and other membrane structures. It has been found that drebrin plays a major role in neuronal development and synaptic plasticity. ${ }^{12}$

Thus, we hypothesize that these cell membrane-related molecules may have potential role in neurodegeneration. The main objective of the present study was to determine NKA, SPTA and drebrin in erythrocyte membranes of neurodegenerative patients to verify whether these protein validations in erythrocytes could provide a clue for identifying various neurodegenerative conditions associated with common clinical features that are either movement dysfunction or memory loss.

\section{Materials and methods}

\section{Study subjects/blood sample collection}

The present case-control study design was approved by the Madras Medical College, Chennai, India (Institutional
Ethical Committee number 38122012; the approval was granted by the committee at a meeting held on December 11, 2012 at the Madras Medical College, Chennai, India). Subjects in the age group of 25-60 years, diagnosed with neurodegenerative condition $(n=200)$ and enrolled at the Department of Neurology and Neuroscience at the Government General Hospital, Chennai, were recruited for the present study. Age-matched $(n=120)$ individuals who were normal and healthy were recruited as control subjects in the study. On our request, clinicians (neurologists) calculated the MBRS score for all the patients. Peripheral blood samples $(5 \mathrm{~mL})$ were collected in a heparinized vacutainers (BD, Franklin Lakes, NJ, USA) with a prior informed consent to the recruited individuals. All the blood samples were transported, at ambient temperature, immediately to the Department of Biochemistry, University of Madras, Guindy Campus, Chennai, and processed. All participants provided written informed consent to participate in the study.

\section{Inclusion criteria}

The diagnosed complaints were as follows: PD ( $\mathrm{n}=76)$, ALS $(n=68)$, ataxia $(n=60)$ and dementia $(n=25)$. Patients who were untreated and/or treated (PD patients were administered with syndopa and ALS patients with riluzole) for a period of 1 week were included. The patients were clinically confirmed by the neurologist. The clinical data are provided in Table 1.

\section{Exclusion criteria}

Patients with cerebrovascular disease, endocrine disorders, diabetes, cancer, anemia and also patients with smoking and drinking habits and/or drug addiction were excluded.

\section{Preparation of erythrocyte membrane}

Erythrocyte membranes were prepared by the method described by Matteucci et $\mathrm{al}^{13}$ with some modifications. The membrane preparation was carried out at $4^{\circ} \mathrm{C}$. Briefly, erythrocytes from $5 \mathrm{~mL}$ of fresh venous blood were separated after diluting (1:1) the blood with sterile phosphate-buffered saline (PBS). Mononuclear white blood cells were separated from red cells by Ficoll (Histopaque 1077; Sigma-Aldrich Co., St.Louis, MO, USA) density gradient centrifugation. In order to remove the Ficoll residue, red blood cells were washed three times with PBS. Packed erythrocytes were washed twice in $20 \mathrm{~mL}$ of an isotonic medium, $\mathrm{pH} \mathrm{7.4,}$ containing 0.25 M mannitol, $20 \mathrm{mM}$ Tris, and $1 \mathrm{mM}$ EDTA. The pellet was resuspended in $2 \mathrm{~mL}$ of the aforementioned medium. After the addition of $18 \mathrm{~mL}$ of deionized water, the 
Table I Mean of age, sex ratio and clinical manifestation of ND subjects

\begin{tabular}{|c|c|c|c|c|c|}
\hline & Control & PD & ALS & Ataxia & Dementia \\
\hline Mean age, years & $42.31 \pm 3.48$ & $48.80 \pm 3.07$ & $42.5 I \pm 2.8 I$ & $31.28 \pm 3.26$ & $65.18 \pm 3.33$ \\
\hline Sex (male: female) & $74: 46$ & $42: 30$ & $40: 24$ & $32: 18$ & $6: 3$ \\
\hline Age range (years) & $30-63$ & $29-68$ & $25-62$ & $25-57$ & $57-78$ \\
\hline Clinical manifestations & & $\begin{array}{l}\text { a) Resting tremor } \\
\text { b) Sleep disturbance } \\
\text { c) Lethargy }\end{array}$ & $\begin{array}{l}\text { Limb-onset ALS with } \\
\text { lower motor neuron } \\
\text { signs in the limbs }\end{array}$ & $\begin{array}{l}\text { a) Gait } \\
\text { b) Tremor } \\
\text { c) Limbic dysfunction }\end{array}$ & Mild cognitive impairment \\
\hline
\end{tabular}

Notes: \#Values are expressed as mean $\pm \mathrm{SE}$.

Abbreviations: ND, neurogenerative disorder; PD, Parkinson's disease; ALS, amyotrophic lateral sclerosis; SE, standard error.

suspension was stirred on ice for 15 minutes and centrifuged at $20,000 \times g$ for 20 minutes. The pellet was resuspended in $2 \mathrm{~mL}$ of mannitol-Tris-EDTA buffer, stirred for 15 minutes after the addition of $18 \mathrm{~mL}$ of deionized water, and centrifuged at 20,000 $\times g$ for 20 minutes. The last procedure was repeated twice. Ghost membranes were suspended in $0.5 \mathrm{~mL}$ of $40 \mathrm{mM}$ Tris- $\mathrm{HCl}$ buffer, $\mathrm{pH} \mathrm{7.4,} \mathrm{to} \mathrm{obtain} \mathrm{a} \mathrm{final} \mathrm{protein}$ concentration of $0.8-1.5 \mathrm{mg} / \mathrm{mL}$. Erythrocyte membrane thus obtained was used for the evaluation of NKA activity.

\section{Determination of NKA's activity}

NKA's activities were assayed according to the procedure described by Tsakiris and Deliconstantinos. ${ }^{14}$ Ten microliters of isolated erythrocyte membrane was added to the reaction mixture containing $5.0 \mathrm{mM} \mathrm{MgCl}_{2}, 80.0 \mathrm{mM} \mathrm{NaCl}, 20.0 \mathrm{mM}$ $\mathrm{KCl}, 40.0 \mathrm{mM}$ Tris- $\mathrm{HCl}$ buffer, $\mathrm{pH} 7.4$, and made up to a final volume of $0.2 \mathrm{~mL}$. The reaction was started by the addition of ATP (vanadium-free disodium salt) to obtain a final concentration of $3.0 \mathrm{mM}$. The reaction was stopped after 10 minutes by the addition of $0.2 \mathrm{~mL}$ of $0.66 \mathrm{M}$ trichloroacetic acid. NKA activity was calculated by the difference between the two values. Released inorganic phosphate ( $\mathrm{Pi}$ ) was measured by the method of Fiske and Subbarow. ${ }^{15}$ Results were expressed as $\mathrm{nmol} \mathrm{Pi} / \mathrm{min} / \mathrm{mg}$ protein. Protein was measured by the method of Bradford, using bovine serum albumin as standard. All assays were run in triplicate.

\section{Western blot of NKA, SPTA and drebrin}

The cells were lysed using RIPA buffer and then were separated by sodium dodecyl sulfate-polyacrylamide gel electrophoresis and transferred to nitrocellulose membranes. The transblotted membranes were incubated with goat antiNKA $\alpha_{2}$ (1:500 dilution; Santa Cruz Biotechnology Inc., Dallas, TX, USA), anti-SPTA (1:500 dilution; Santa Cruz Biotechnology Inc.), and anti-drebrin (1:500 dilution; Santa Cruz Biotechnology Inc). The blots were then washed with PBS and incubated with the horseradish-peroxidase-linked secondary antibody (Merck, Kenilworth, NJ, USA) and detected using 3, 3 '-diaminobenzidine (Sisco Research Laboratories Pvt. Ltd., Mumbai, India).The band intensities were further measured using ImageJ Editor, their relative densities were calculated, and analysis of variance (ANOVA) was performed using the triplicate values of the relative density.

\section{Isolation of RNA for qRT PCR}

Total RNA was extracted from the whole blood using TRIzol reagent (category number 15596026; Thermo Fisher Scientific, Waltham, MA, USA). Real-time PCR was performed to measure the expression of $\mathrm{NKA} \alpha_{2}, \mathrm{NKA} \alpha_{3}$, $\alpha$-spectrin and drebrin using SYBER Green mastermix (Takara, Shiga, Japan) with CFX96 real-time PCR system (Bio-Rad Laboratories, Inc., Hercules, CA, USA). The sequence for $N K A \alpha_{2}: 5^{\prime}$-CTTTGCATTGACCTGGGCAC-3', antisense 5'-TTGTCCGTCTGGGAGTTTCG-3'; NKAa3: 5'-CAGGAGAGCCACGGACAAGAA-3', antisense 5'-TTGTCCGTCTGGGAGTTTCG-3'; aspectrin: $5^{\prime}$-GGCTGATGGGATTCTAGGAC-3', antisense 5'-AACCTGGCAAGATAAAATGTGTC-3'; drebrin: 5'-ACTCAAAAGGAGGGGACCCA-3', antisense 5'-TACAGGAGGCGGAACCTTTG-3'. GAPDH gene was used as an internal control using the sense primer 5'-GCACCGTCAAGGCTGAGAAC-3' and antisense primer 5'-TGGTGAAGACGCCAGTGGA-3' (primers were obtained from Sigma-Aldrich Co.). The comparative threshold (CT) cycle values were derived from qbasePLUSsoftware. The $\Delta \mathrm{CT}$ method of relative quantification was used to determine the fold changes in expression $(\Delta \mathrm{CT}=\Delta \mathrm{CT}$ reference $-\Delta \mathrm{CT}$ target $)$.

\section{Statistical analysis}

Unpaired Student's $t$-test was used to compare the mean fluorescence intensity and the fold change in the samples and the $p$-value $\leq 0.05$ was considered as significant. One-way ANOVA was also carried out to compare the variation among the groups and significant values were represented. Nonpara- 
metric correlation was performed to study the comparison between age, enzyme activity and movement dysfunction. The statistical analyses were carried out and graphs were generated using Graph Pad PRISM 5.0.

\section{Results}

\section{Estimation of NKA in erythrocytes}

The activity of NKA, an active ion transporter located in the erythrocyte membrane, was found to be decreased in ND patients when compared to that of control $(p \leq 0.05)$. Further, comparative assessment among the ND subjects showed an increase in the activity of NKA in PD and dementia while a reduced activity was encountered in ALS and ataxia (Figure 1). Correlation analysis was performed for movement dysfunction in PD, ALS and ataxia and patients were scored for MBRS from zero to three. The Spearman's correlation analysis showed that the activity of NKA was significantly correlated with MBRS in PD and ataxia patients, whereas there was no significant correlation in ALS. The correlation of activity of NKA with age did not show any significant change, the details of which are given in Table 2 .

\section{Western blot}

On analysis of the protein expression, there was a significant decrease in the levels of SPTA, drebrin and NKA $\alpha_{2}$ in ND subjects $(p<0.05)$ when compared to control. In case of ALS, the expression of SPTA and drebrin was found to be diminishing (Figure 2).

Table 2 Correlation analysis of NKA activity in ND

\begin{tabular}{lll}
\hline & MBRS & Age \\
\hline PD & $r=0.5367, p=0.0293^{*}$ & NS \\
ALS & $r=0.09759, p=0.3756$ & NS \\
Ataxia & $r=0.6596, p=0.007 I^{* *}$ & NS \\
\hline
\end{tabular}

Notes: Nonparametric correlation (Spearman's rho, $r$ ) was used to correlate clinical features (movement dysfunction in accordance with MBRS and age) and NKA activity in ND subjects with movement disorders; * $p \leq 0.05$ indicates significance; $* * p \leq 0.01$ indicates highly significant.

Abbreviations: ND, neurogenerative disorder; PD, Parkinson's disease; ALS, amyotrophic lateral sclerosis; MBRS, Modified Bradykinesia Rating Scale; NKA, $\mathrm{Na}^{+}$I $\mathrm{K}^{+}$-ATPase; NS, nonsignificant.
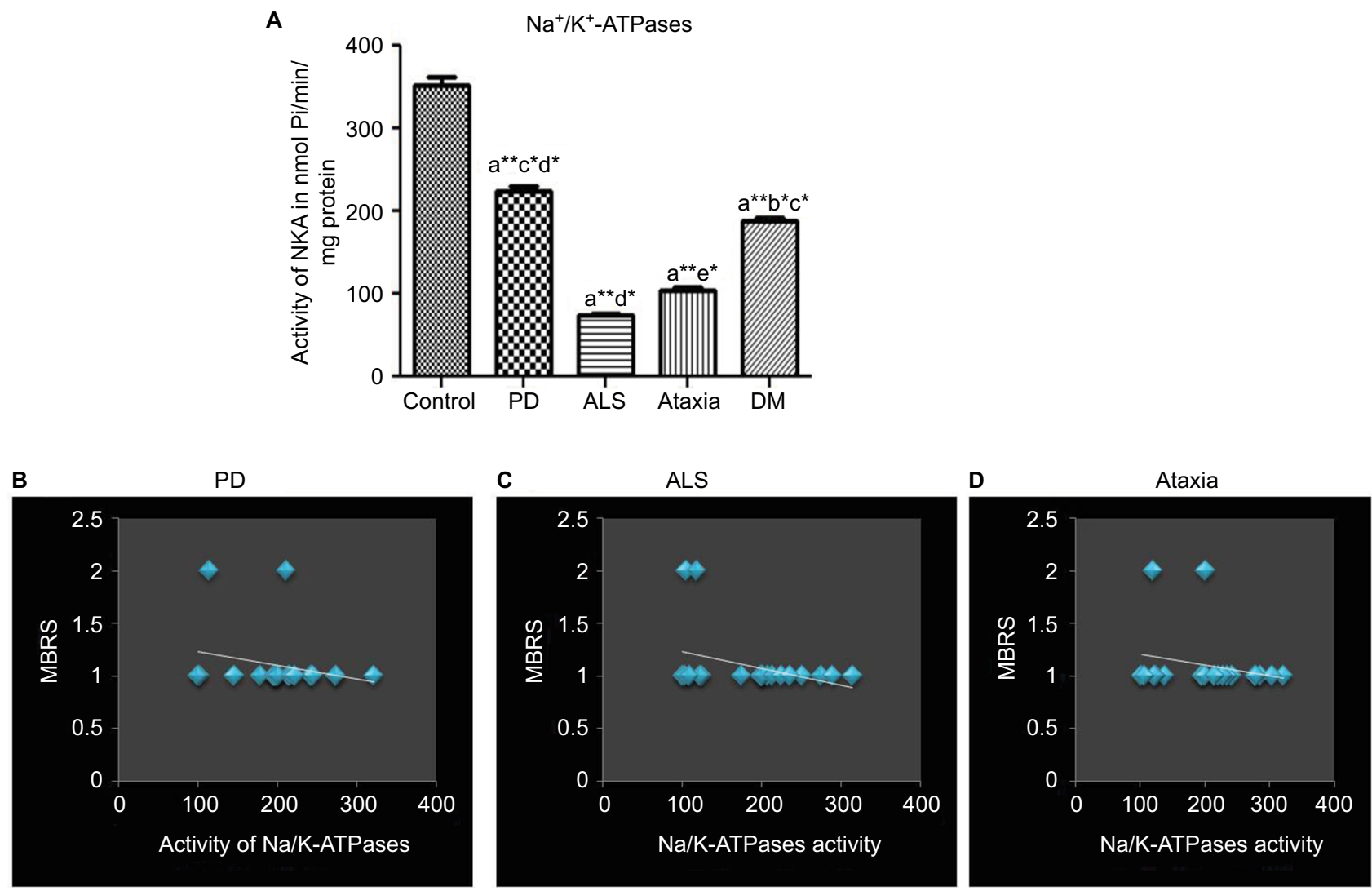

Figure I (A) The representative histogram of the activity of NKA activity of control and the recruited neurodegenerative subjects. ANOVA was performed ( $p \leq 0.05$ ) compared with (a) control, (b) Parkinson's disease, (c) amyotrophic lateral sclerosis, (d) ataxia and (e) dementia. Error bars indicate SEM; (B) data on the linear correlation analysis between MBRS and Parkinson's disease; (C) data on the linear correlation analysis between MBRS and amyotrophic lateral sclerosis; (D) data on the linear correlation analysis between MBRS and ataxia. Nonparametric correlation (Spearman's rho, $r$ ) was used to correlate clinical features (movement dysfunction in accordance with MBRS and age) and NKA activity in ND subjects with movement disorders; ${ }^{*} p \leq 0.05$ indicates significance; ** $p \leq 0.0$ I indicates highly significant.

Abbreviations: ANOVA, analysis of variance; PD, Parkinson's disease; ALS, amyotrophic lateral sclerosis; DM, dementia; SEM, standard error of the mean; MBRS, Modified Bradykinesia Rating Scale; NKA, Na ${ }^{+} / \mathrm{K}^{+}$-ATPase; ND, neurogenerative disorder. 
A Control PD ALS Ataxia DM

$\alpha-$ Spectrin

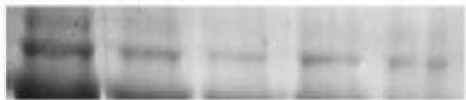

$\mathrm{Na}^{+} / \mathrm{K}^{+}$-ATPase $\alpha_{2}$

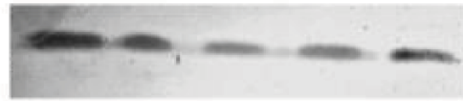

Drebrin

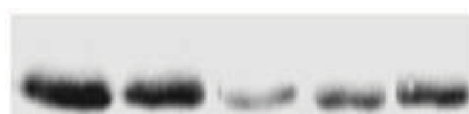

$\beta$-Tubulin

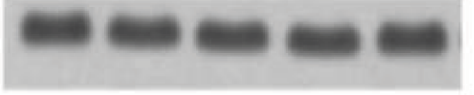

B

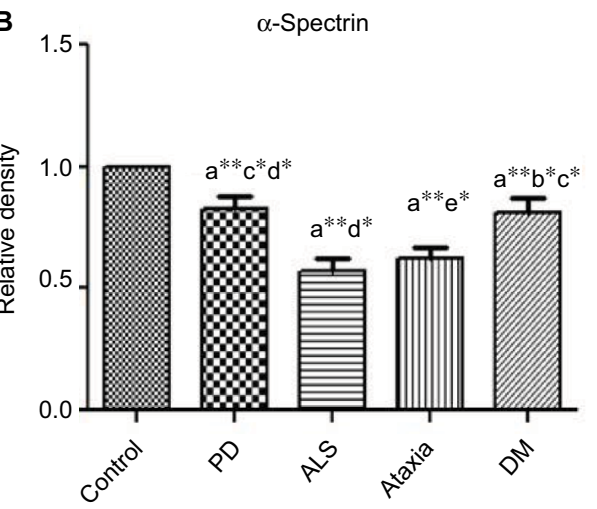

Control

$\mathbf{D P D}$

目ALS

血 Ataxia

표 DM

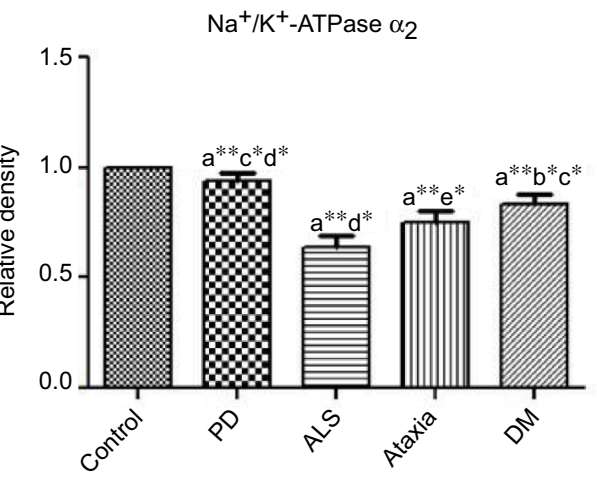

网 Control

$\mathbf{B P D}$

回ALS

핑 Ataxia

매 DM

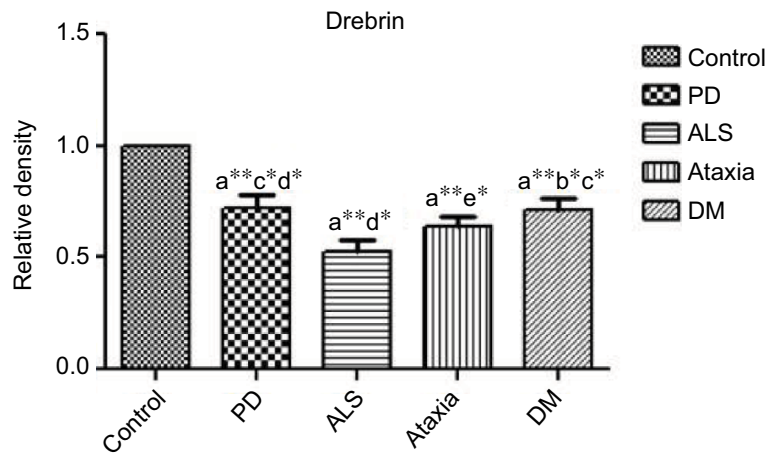

Figure 2 (A) Representative Western blot results (experiments were performed in triplicate); (B) relative density graphs of mean \pm SD. ANOVA was performed ( $p \leq 0.05$ ) compared with (a) control, (a) Parkinson's disease, (a) amyotrophic lateral sclerosis, (a) ataxia, (a) dementia. Error bars indicate SEM. * $p \leq 0.05$ indicates significance; ** $p \leq 0.01$ indicates highly significant.

Abbreviations: ANOVA, analysis of variance; SEM, standard error of the mean; PD, Parkinson's disease; ALS, amyotrophic lateral sclerosis; DM, dementia. 

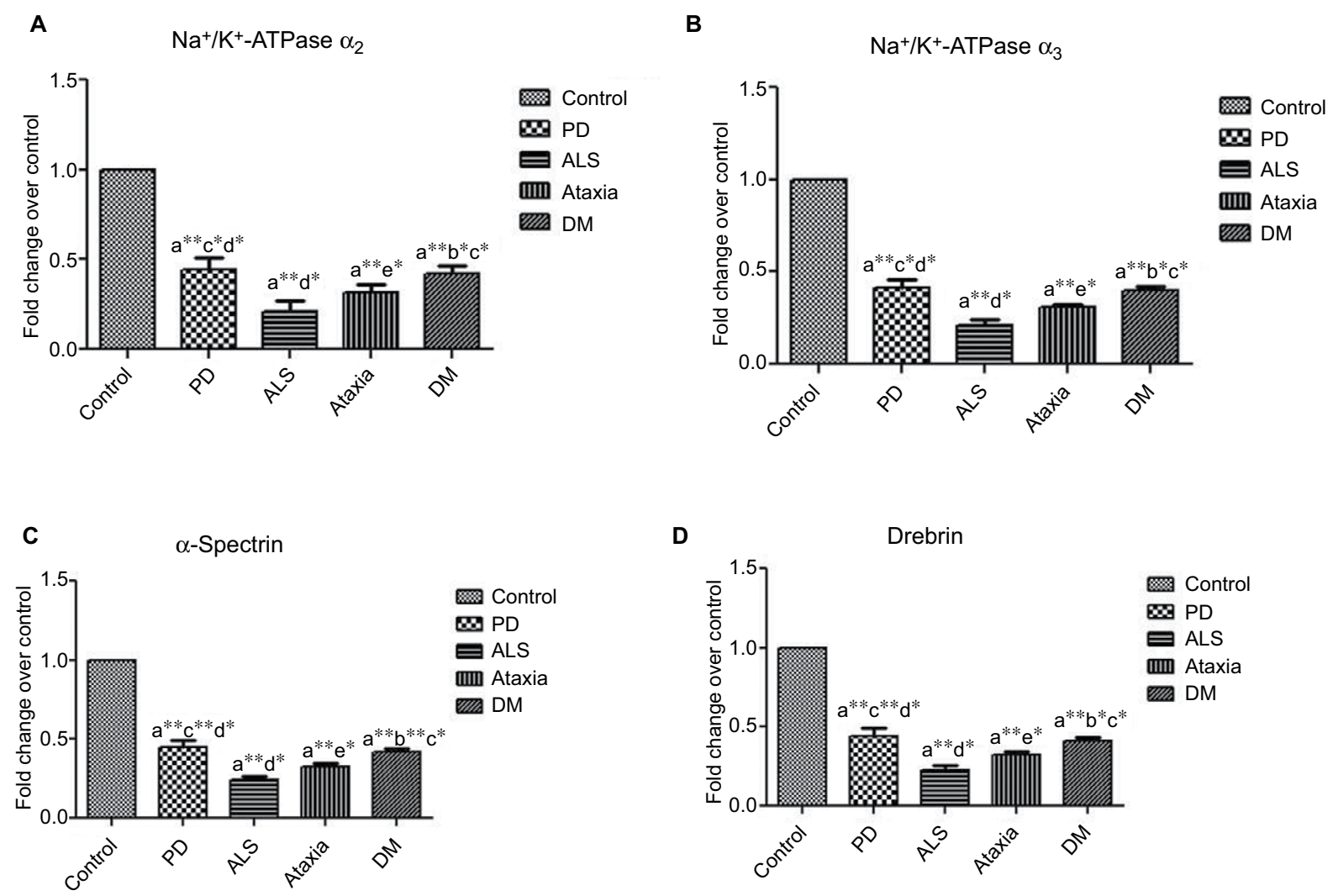

Figure 3 Data on qRT PCR analysis between control and neurodegenerative subjects: (A) NKA $\alpha_{2}$, (B) NKA $\alpha_{3}$, (C) SPTA and (D) drebrin. ANOVA was performed using the mean fold change ( $p \leq 0.05$ ) compared with (a) control, (b) Parkinson's disease, (c) amyotrophic lateral sclerosis, (d) ataxia and (e) dementia. Error bars indicate SEM. * $p \leq 0.05$ indicates significance; $* * 00.01$ indicates highly significant.

Abbreviations: ANOVA, analysis of variance; SEM, standard error of the mean; NKA, $\mathrm{Na}^{+} / \mathrm{K}^{+}-\mathrm{ATPase}$; SPTA, $\alpha$-spectrin; PD, Parkinson's disease; ALS, amyotrophic lateral sclerosis; DM, dementia.

\section{qRT PCR}

Analysis of $\mathrm{NKA}_{2}, \mathrm{NKA}_{3}$, SPTA and drebrin mRNA levels showed a significant decrease in the fold of expression in NDs, unlike in the healthy subjects. The expression levels of both SPTA and drebrin were found to be the least in case of ALS. The graphical representation of fold change is depicted in Figure 3).

\section{Discussion}

The observation of reduction in the activity of NKA can be regarded as a preliminary phase showing neuronal vulnerability as studies have reported that deregulation and deficiency of NKA activity can be one of the major implications in neurodegeneration, since it helps in maintaining the ionic gradient of the cells, thereby regulating cellular volume and preventing the swelling and lysis of cells such as neurons. ${ }^{16}$ Thus, we suggest that the changes in the level of NKA activity can be an admonition to indicate the ongoing process of neuronal degeneration. On correlating the clinical features with the activity of NKA, we could observe that there was a significant correlation with the activity of NKA and MBRS in patients with PD and ataxia, and this finding is in accordance with the report which has shown that misbalance of ionic concentration in basal ganglia and cortical motor neuron can result in dystonia and bradykinesia. ${ }^{17}$ Bradykinesia is one of the common clinical features among PD, ALS and ataxia, and hence we chose to study the severity of this clinical feature with NKA. Our findings also correlate with a report showing that rapid-onset dystonia parkinsonism mutation in NKA was the primary cause and that this rare genetic neurological disorder has an abrupt onset with the development of bradykinesia and postural instability. ${ }^{18}$ Thus, we would like to hypothesize that NKA can give an early indication of these NDs.

There are three different isoforms of NKA based on the $\alpha$ subunit: $\alpha_{1}, \alpha_{2}, \alpha_{3}$. We observed a considerable difference in the expression of these NKA isoforms among different NDs. Since the isoform NKA $\alpha_{2}$ was repored to be similar in both brain and erythrocyte, ${ }^{19}$ we chose to study this protein in these NDs. Further, an in vitro study has shown that neuronal NKA inhibition could be achieved by dopamine and its oxidation products; this inactivation of NKA by dopamine can lead to various toxic sequelae eventually causing dopaminergic cell death in PD. ${ }^{16}$ So, we suggest that treatment targeted to 
restore the ionic imbalance in the case of NDs, such as PD, may help to delay the progression of disease.

We have observed that SPTA can be an important parameter to diagnose ALS at early stages of the disease, as there was a severe reduction in their expression level. Studies have reported that mutation in SPTA induced destabilization and dislocation of membrane receptors and can lead to neurodegeneration. ${ }^{20} \mathrm{RBC}$ membranes partially devoid of a spectrin-actin network can no longer be present as a ghost, but start to fragment and appear as small, $50 \mathrm{~nm}$ reversed vesicles. The importance of spectrin can be observed mainly in structure which is highlighted by the fact that mutations in genes encoding or regulating the expression of particular skeletal components underlie the molecular mechanism. ${ }^{21}$

The reduction in the level of drebrin in patients with ALS and dementia can be an indicative of an apparent impairment in cognition in these neurodegenerative conditions which was not encountered in PD and ataxia. As drebrin acts along with actin in neurite processes in post-migratory neurons, their decreased levels can affect the cell processes by deregulation of actin filament formation. ${ }^{22}$ Associating our observation of abnormality in drebrin with Yan et al, which reported drebrin is important in the degeneration of dendritic tree at the cortex and relates with impairment in cognitive responses, ${ }^{8}$ we also suggest that the decreased level of drebrin in the blood of patients with NDs at an early stage can be an useful indicator.

Though there have been extensive studies on the identification of etiology and diagnosis of ND, the research still remains incomplete. The compromising of blood-brain barrier has also been implicated in NDs and may also contribute to a cycle of changes that serve to aggravate cell loss. ${ }^{23}$ Thus, we suggest that alterations in the erythrocyte membrane obtained from the blood of patients with NDs provide a suggestion for faster diagnosis. Our findings look forward to assist the clinicians in the symptomatic identification of neurodegeneration in patients along with their scoring and characterization.

\section{Acknowledgment}

We gratefully acknowledge the financial support from the University Grant Commission - Basic Scientific Research (award number GCCO/A-2/UGC-MERITORIOUS /2012/1; dated April 2, 2012).

\section{Disclosure}

The authors report no conflicts of interest in this work.

\section{References}

1. Francois JG, Vingerhoets, Schulzer M, Calne DB, Snow BJ. Which clinical sign of Parkinson's disease best reflects the nigrostriatal lesion? Ann Neurol. 1997;41(1):58-64.

2. Kishore A, Espay AJ, Marras C, et al. Unilateral versus bilateral tasks in early asymmetric Parkinson's disease: differential effects on bradykinesia. Mov Disord. 2007;22(3):328-333.

3. Berardelli A, Rothwell JC, Thompson PD, Hallett M. Pathophysiology of bradykinesia in Parkinson's disease. Brain. 2001;124(Pt 11):2131-2146.

4. Molochnikov L, Rabey JM, Dobronevsky E, et al. A molecular signature in blood identifies early Parkinson's disease. Mol Neurodegener. 2012;7:26.

5. Ghezzo A, Visconti P, Abruzzo PM, et al. Oxidative stress and erythrocyte membrane alterations in children with autism: correlation with clinical Features. PLoS One. 2013;8(6). doi:10.1371/journal.pone.0066418.

6. Bedin M, Estrella CH, Ponzi D, et al. Reduced Na(+), K(+)-ATPase activity in erythrocyte membranes from patients with phenylketonuria. Pediatr Res. 2001;50(1):56-60:e66418.

7. Goodman SR, Krebs KE, Whitfield CF, Riederer BM, Zagon IS. Spectrin and related molecules. CRC Crit Rev Biochem. 1988;23(2):171-234.

8. Yan X-X, Jeromin A, Jeromin A. Spectrin breakdown products (SBDPs) as potential biomarkers for neurodegenerative diseases. Curr Transl Geriatr Exp Gerontol Rep. 2012;1(2):85-93.

9. Lorenzo DN, Li MG, Mische SE, Armbrust KR, Ronum LPW, Hays TS. Spectrin mutations that cause spinocerebellar ataxia type 5 impair axonal transport and induce neurodegeneration in Dmsophila. J Cell Biol. 2010;189(1):143-158.

10. Shim KS, Lubec G. Drebrin, a dendritic spine protein, is manifold decreased in brains of patients with Alzheimer's disease and Down syndrome. Neurosci Lett. 2002;324(3):209-212.

11. Asada H, Uyemura K, Shirao T. Actin-binding protein, drebrin, accumulates in submembranous regions in parallel with neuronal differentiation. J Neurosci Res. 1994;38(2):149-159.

12. Butkevich E, Hülsmann S, Wenzel D, Shirao T, Duden R, Majoul I. Drebrin is a novel connexin-43 binding partner that links gap junctions to the submembrane cytoskeleton. Curr Biol. 2004;14(8):650-658.

13. Matteucci E, Cocci F, Pellegrini L, Gregori G, Giampietro O. Measurement of ATPases in red cells: setting up and validation of a highly reproducible method. Enzyme Protein. 1993;48(2):105-119.

14. Tsakiris S, Deliconstantinos G. Influence of phosphatidylserine on $\mathrm{Na}+$ $+\mathrm{K}+$ )-stimulated ATPase and acetylcholinesterase activities of dog brain synaptosomal plasma membranes. Biochem J. 1984;220(1):301-307.

15. Fiske $\mathrm{CH}$, Subbarow Y. The colorimetric determination of phosphorus. J Biol Chem. 1925;66:375-400.

16. Arnaiz GR de L, Ordieres MGL. Brain Na+, K+-ATPase activity in aging and disease. Int J Biomed Sci. 2014;10(2):85-102.

17. Brashear A, Dobyns WB, De Carvalho Aguiar P, et al. The phenotypic spectrum of rapid-onset dystonia-parkinsonism (RDP) and mutations in the ATP1A3 gene. Brain. 2007;130(3):828-835.

18. De Carvalho Aguiar P, Sweadner KJ, Penniston JT, et al. Mutations in the $\mathrm{Na}+\mathrm{K}+-\mathrm{ATPase}$ ??3 gene ATP1A3 are associated with rapid-onset dystonia parkinsonism. Neuron. 2004;43(2):169-175.

19. Shamraj OI, Lingrel JB. A putative fourth $\mathrm{Na}+, \mathrm{K}(+)$-ATPase alpha-subunit gene is expressed in testis. Proc Natl Acad Sci USA. 1994;91(26): 12952-12956.

20. Czogalla A, Sikorski AF. Spectrin and calpain: a "target" and a "sniper" in the pathology of neuronal cells. Cell Mol Life Sci. 2005;62(17): 1913-1924.

21. MacHnicka B, Grochowalska R, Bogusławska DM, Sikorski AF, Lecomte MC. Spectrin-based skeleton as an actor in cell signaling. Cell Mol Life Sci. 2012;69(2):191-201.

22. Hayashi K, Shirao T. Change in the shape of dendritic spines caused by overexpression of drebrin in cultured cortical neurons. $J$ Neurosci. 1999;19(10):3918-3925.

23. Palmer AM. The role of the blood brain barrier in neurodegenerative disorders and their treatment. J Alzheimer's Dis. 2011;24(4):643-656. 


\section{Publish your work in this journal}

Degenerative Neurological and Neuromuscular Disease is an international, peer-reviewed, open access journal focusing on research into degenerative neurological and neuromuscular disease, identification of therapeutic targets and the optimal use of preventative and integrated treatment interventions to achieve improved outcomes, enhanced survival and quality of life for the patient. The manuscript management system is completely online and includes a very quick and fair peer-review system. Visit http://www.dovepress.com/testimonials.php to read real quotes from published authors.

Submit your manuscript here: https://www.dovepress.com/degenerative-neurological-and-neuromuscular-disease-journal 